\title{
Primer registro de Compsopogon caeruleus (Rhodophyta, Compsopogonales) en Córdoba (Argentina) y consideraciones sobre la presencia del género en Argentina
}

First record of Compsopogon caeruleus (Rhodophyta, Compsopogonales) in Córdoba (Argentina) and considerations on the genus in Argentina

D.0.I.: doi.org/10.30550/j.lil/2018.55.2/6

Leyes, Claudia ${ }^{1,2^{*}}$; Alejandra Bustamante ${ }^{1}$; Cecilia Giovanola ${ }^{1}$; Claudia Daga ${ }^{2,3}$

1 Programa de Investigación sobre los Ecosistemas Acuáticos, Centro de Tecnología de Uso del Agua, Instituto Nacional del Agua (INA). Av. Ambrosio Olmos 1142, 2do piso, (5000) Córdoba, Argentina.

a Cátedra de Diversidad Biológica I. Facultad de Ciencias Exactas Físicas y Naturales. Universidad Nacional de Córdoba. Avenida Vélez Sarsfield 299, (5000) Córdoba, Argentina.

3 Área de Proyectos Especiales. Facultad de Ciencias Exactas Físicas y Naturales. Universidad Nacional de Córdoba. Avenida Vélez Sarsfield 299, (5000) Córdoba, Argentina.

* Autor corresponsal: ca_leyes@yahoo.com.ar

- Resumen - La Rhodophyta Compsopogon caeruleus es registrada por primera vez en distintos ambientes de la provincia de Córdoba. El material fue recolectado entre los años 2012 y 2016, en un lago artificial urbano, un arroyo serrano y un río con características de llanura. Se describe, dibuja, fotografía, se presenta un mapa de su distribución y se compara con otras especies citadas en Argentina.

Palabras clave: Alga roja; arroyo; lago; río.

- Abstract - The Rhodophyta Compsopogon caeuruleus, new record for Córdoba (Argentina). The material was collected between 2012 and 2016, from an artificial urban lake, mountain stream and river of plain. Description, illustration, photos, distribution map of the species are provided, and compared with other species cited in Argentina.

Keywords: Red algae; stream; river; lake.

\footnotetext{
> Ref. bibliográfica: Leyes, C.; Bustamante, A.; Giovanola, C.; Daga, C. 2018. Primer registro de Compsopogon caeruleus (Rhodophyta, Compsopogonales) en Córdoba (Argentina) y consideraciones sobre la presencia del género en Argentina. Lilloa 55 (2): 67-74.

> Recibido: 30/05/18 - Aceptado: 29/08/18

$>$ URL de la revista: http://lilloa.lillo.org.ar 


\section{INTRODUCCIÓN}

La mayoría de las algas rojas (Rhodophyta) son marinas, hay alrededor de 7.000 especies descriptas (Guiry, 2012) y sólo el tres por ciento de ellas se encuentra en hábitats dulceacuícolas (Wehr y Sheath, 2003). En Argentina son escasos los trabajos dedicados al estudio de este grupo de algas (Lazo, 1978; Gauna y Parodi, 2005; Daga, Soteras, Daniele, Dominguez, 2014), en especial las referidas a agua dulce. Usualmente, son componentes importantes de la ficoflora de ríos o arroyos, ya sea en términos de abundancia o distribución (Nan et al., 2017), tienen hábitos bentónicos y en comparación poseen menor tamaño que los miembros marinos (Sheath y Hambrook, 1990).

El género Compsopogon pertenece a la familia Compsopogonaceae, dentro del Orden Compsopogonales (Guiry y Guiry, 2018). Aunque este género posee amplia distribución mundial, es más común en regiones tropicales y subtropicales, localizándose ocasionalmente en zonas templadas (Krishnamurthy, 1962; Necchi, Góes, Dip, 1990; Sheath y Hambrook, 1990; Vis, Sheath, Cole, 1992; Rintoul, Sheath, Vis, 1999). Se lo ha identificado en hábitats diferentes, como sistemas lóticos de aguas claras y bajo contenido de nutrientes (Sheath y Hambrook, 1990; Liu y Wang, 2004), aunque también se lo ha observado en aguas con materia orgánica (Sabater, Aboal, Cambra, 1989; Carmona Jiménez y Beltrán Magos, 2007). De la misma manera puede hallarse en sistemas lénticos con niveles elevados de eutrofización (Necchi, 1989; Ceschin et al., 2013; Leyes, 2016) o incluso en aguas salobres (Krishnamurthy, 1962; Sánchez Rodríguez 1974; Necchi, 1989; Ceschin et al., 2013; Leyes, Falco, Daga, 2018).

En Argentina se han citado dos especies, Compsopogon argentinensis en Buenos Aires (Pujals, 1967) y Compsopogon occidentalis en Tucumán (Tracanna, 1979; Taboada, Martínez de Marco, Tracanna, 2016). El objetivo de este trabajo es dar a conocer la presencia de Compsopogon caeruleus en Córdoba, brindar información sobre sus características y distribución, como así también realizar com- paraciones morfológicas entre las especies registradas en el país.

\section{MATERIALES Y MÉTODOS}

Los ejemplares fueron recolectados entre los años 2012 y 2016 procedente de tres sitios (Fig. 1): un lago artificial urbano (Departamento Capital, Lago del Parque Sarmiento, 3125'53'S, 6410'44”O), un arroyo serrano (Departamento Colón, Arroyo Unquillo, $31^{\circ} 14^{\prime} 14^{\prime \prime}$ S, 64²' $8^{\prime} 57^{\prime \prime} \mathrm{O}$ ), y un río con características de llanura (Departamento Calamuchita, Río Tercero, 3209’09”S, $\left.64^{\circ} 08^{\prime} 31^{\prime \prime O}\right)$. El lago del Parque Sarmiento es somero (0,6-1,75 m), de aguas estancadas, baja o nula transparencia, $\mathrm{pH}$ alrededor de 9, salobre, presenta una conductividad mayor a $2.000 \mu \mathrm{S} . \mathrm{cm}^{-1}$ y es eutrófico, con frecuentes floraciones de cianobacterias durante todo el año (Leyes, 2016). El Arroyo Unquillo posee aguas claras y transparentes, escasa profundidad (menor a $1 \mathrm{~m}$ ) y corriente lenta (exceptuando periodos de crecidas estivales). Por último, el Río Tercero es torrentoso de aguas relativamente claras, $\mathrm{pH}$ neutro, conductividad alrededor de 180 $\mu$ S.cm-1 y profundidad variable de acuerdo al sitio considerado, no obstante, los ejemplares fueron obtenidos de la orilla aproximadamente a $30 \mathrm{~cm}$ de profundidad.

El material se obtuvo a partir del raspado de diferentes superficies sumergidas (entre $10-40 \mathrm{~cm}$ de profundidad) tales como rocas, escombros, macrófitas y fue analizado «in vivo» con microscopio óptico. La identificación se realizó consultando a Bourrelly (1970) y los trabajos de: Krishnamurthy (1962), Pujals (1967), Tracanna (1979), Necchi (1989), y Necchi, Branco y Gomes (1999). Luego se fijó con formalina (4\%) e incorporó al herbario del Laboratorio de Hidrobiología de la Facultad de Ciencias Exactas Físicas y Naturales de la Universidad Nacional de Córdoba y al herbario del Programa de Investigación sobre los Ecosistemas Acuáticos del Instituto Nacional del Agua, bajo las siglas LH UNC y PEA-CTUA INA respectivamente, con su numeración correspondiente. 


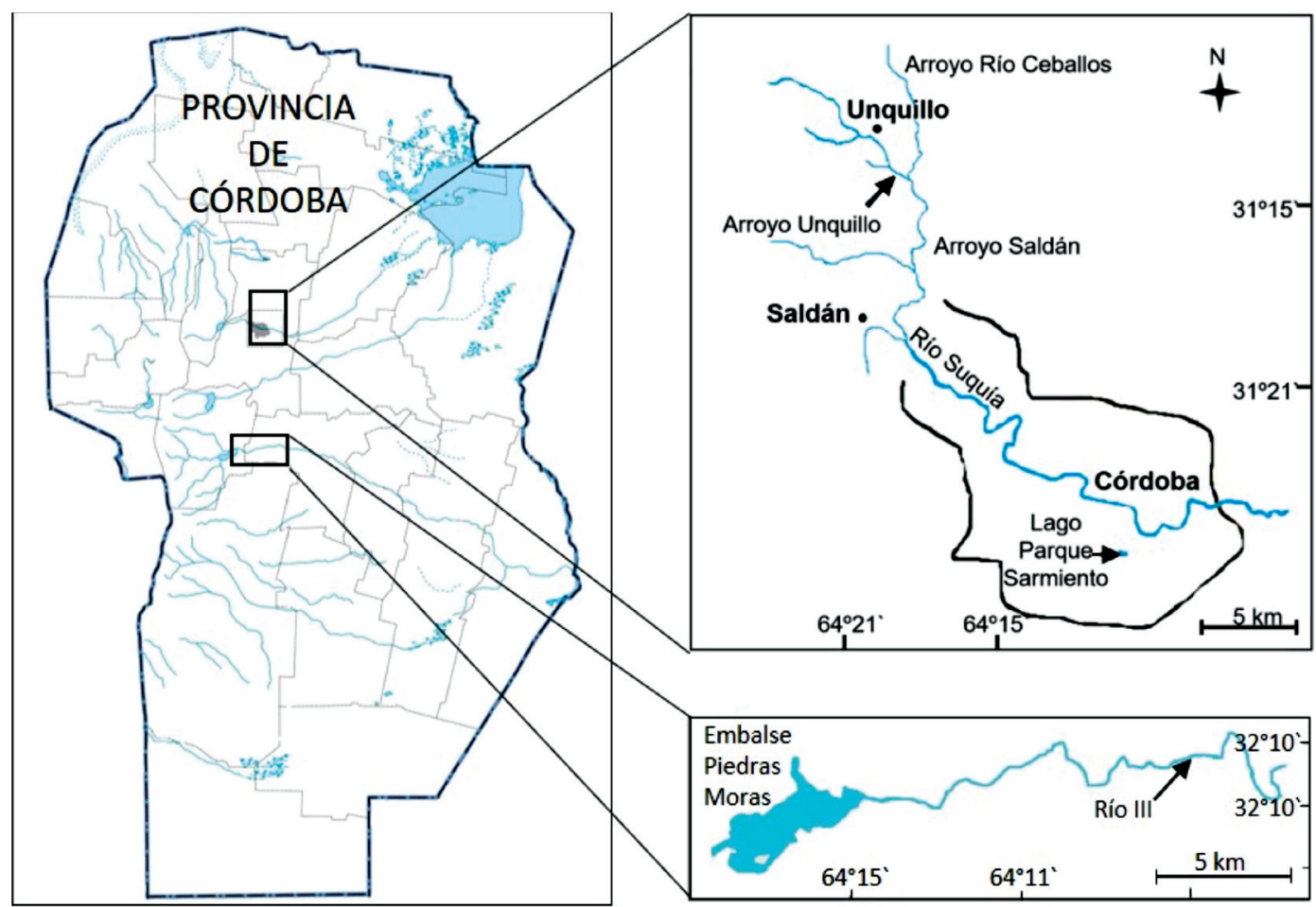

Fig. 1. Ubicación de los sitios donde se registró la presencia de Compsopogon caeruleus (flechas).

\section{RESULTADOS Y DISCUSIÓN}

Compsopogon caeruleus (Balbis ex C. Agardh) Montagne, 1846 (Fig. 2 y 3)

Talo heterótrico, fijado al sustrato mediante rizoides uniseriados que nacen de la base del talo. Uno-varios filamentos erguidos multiseriados, hasta $60 \mathrm{~cm}$ de longitud y $1.500 \mu \mathrm{m}$ de diámetro, constituidos por células centrales rodeadas de células corticales. En las partes viejas presenta constricciones en forma de anillos. De una a dos capas de células corticales, las externas poligonales en vista superficial, 20-57 $\mu \mathrm{m}$ de longitud y 16-37 $\mu \mathrm{m}$ de ancho, las internas presentes sólo en filamentos viejos, globosas, de dimensiones mucho mayores, 140-160 $\mu \mathrm{m}$ de diámetro. Filamentos viejos septados, con células axiales de 160-224 $\mu \mathrm{m}$ de longitud y 160-176 $\mu \mathrm{m}$ de ancho que en algunos casos colapsa, quedando sólo las células corticales. Células de los filamentos uniseriados cilíndricas, 20-29 $\mu \mathrm{m}$ de longitud y 16-20 $\mu \mathrm{m}$ de ancho. Cromatóforos varios, parietales, acintados, cilíndricos, esféricos, irregulares. Color desde verde-oliváceo, verde-grisáceo, hasta azulado. Monosporangios triangulares a esféricos, de 15-18 $\mu \mathrm{m}$ de diámetro. Microsporangios no observados.

\section{HÁBITAT}

Epífito sobre diferentes sustratos. En el lago del Parque Sarmiento sobre rocas, escombros, pequeños moluscos, fragmentos de corteza y raíces de árboles. Los ejemplares fueron de dimensiones intermedias (hasta $15 \mathrm{~cm}$ ) y estaban conviviendo con clorofitas filamentosas como: Ulva flexuosa, Rhizoclonium hieroglyphicum, Oedogonium sp. y Cladophora sp. En el arroyo Unquillo se observó sobre piedras, con una longitud de hasta $60 \mathrm{~cm}$, cohabitando con una rodofita: Batrachospermum atrum var. puiggarianum, una xantoficea: Vaucheria geminata y con clorofitas: Oedogonium sp. y Cladophora sp. No obstante, en Río Tercero se detectó creciendo como epífita de plantas acuáticas de 
A
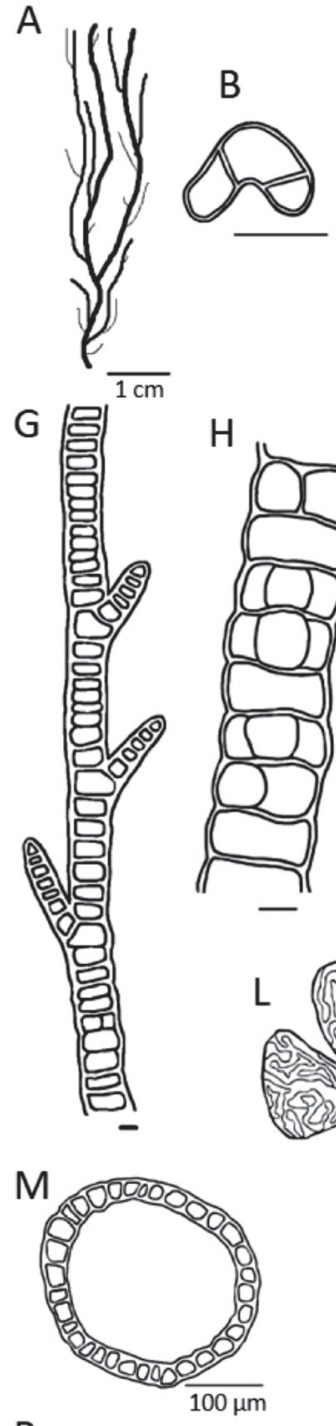

$\mathrm{P}$

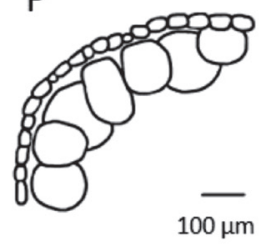

C

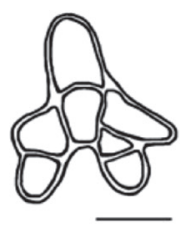

$\mathrm{H}$

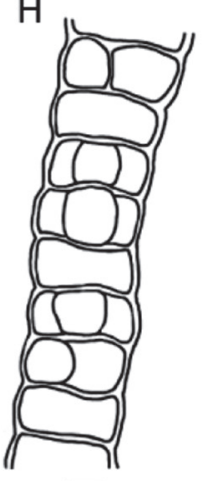

$\mathrm{L}$

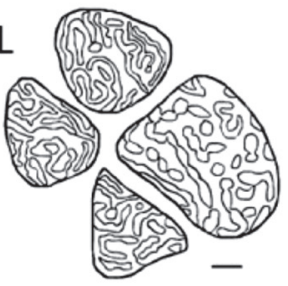

D

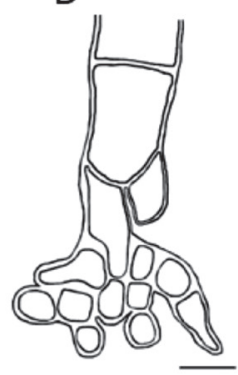

$\mathrm{E}$
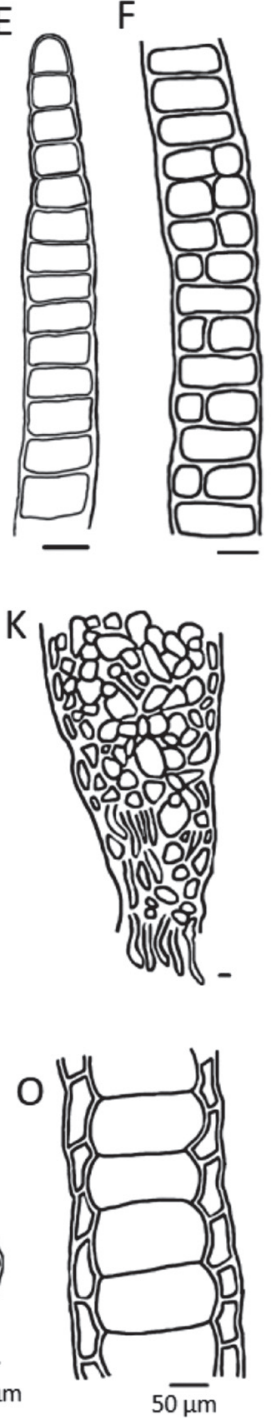
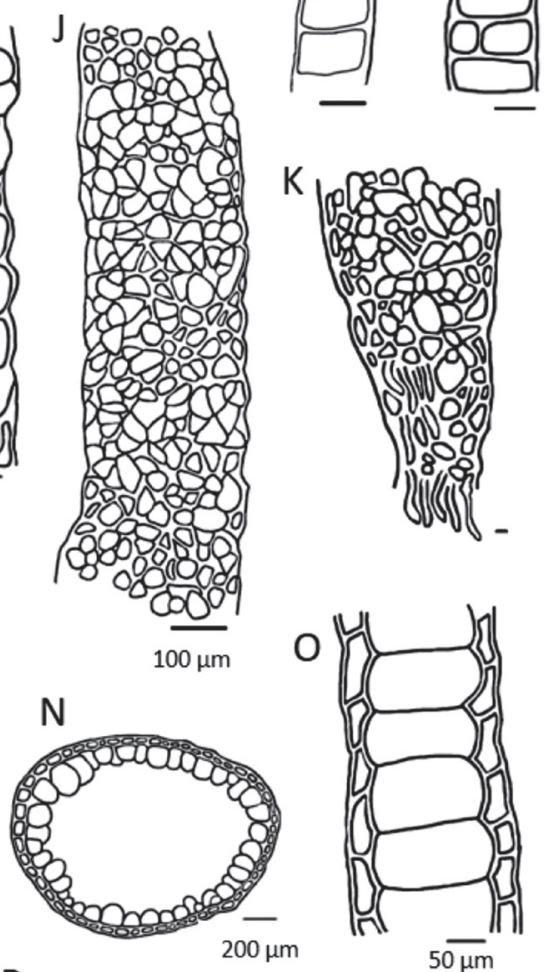

$\mathrm{R}$
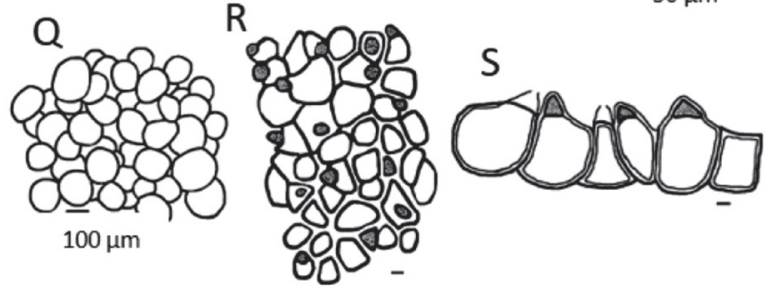

Fig. 2. Compsopogon caeruleus. A] Aspecto general. B) Etapa temprana del desarrollo. C-D] Desarrollo del sistema de fijación al sustrato. E) Filamento uniseriado. F) División celular en el filamento uniseriado. G) Patrón de ramificaciones uniseriadas. H-J) Diferentes estados de formación de la corteza. K) Porción basal del talo con rizoides. L) Células corticales con cromatóforos. M) Corte transversal de filamento con una capa de corteza. N) Corte transversal de filamento con dos capas de corteza. 0) Corte longitudinal de filamento septado donde se observan las células corticales (externas) y las células axiales (internas). P) Detalle del corte transversal con dos capas corticales. Q) Células corticales interiores en vista superficial. R] Vista superficial de células corticales con monosporangios. S] Vista lateral de células corticales con monosporangios. Las escalas corresponden a $10 \mu \mathrm{m}$ salvo indicación. 

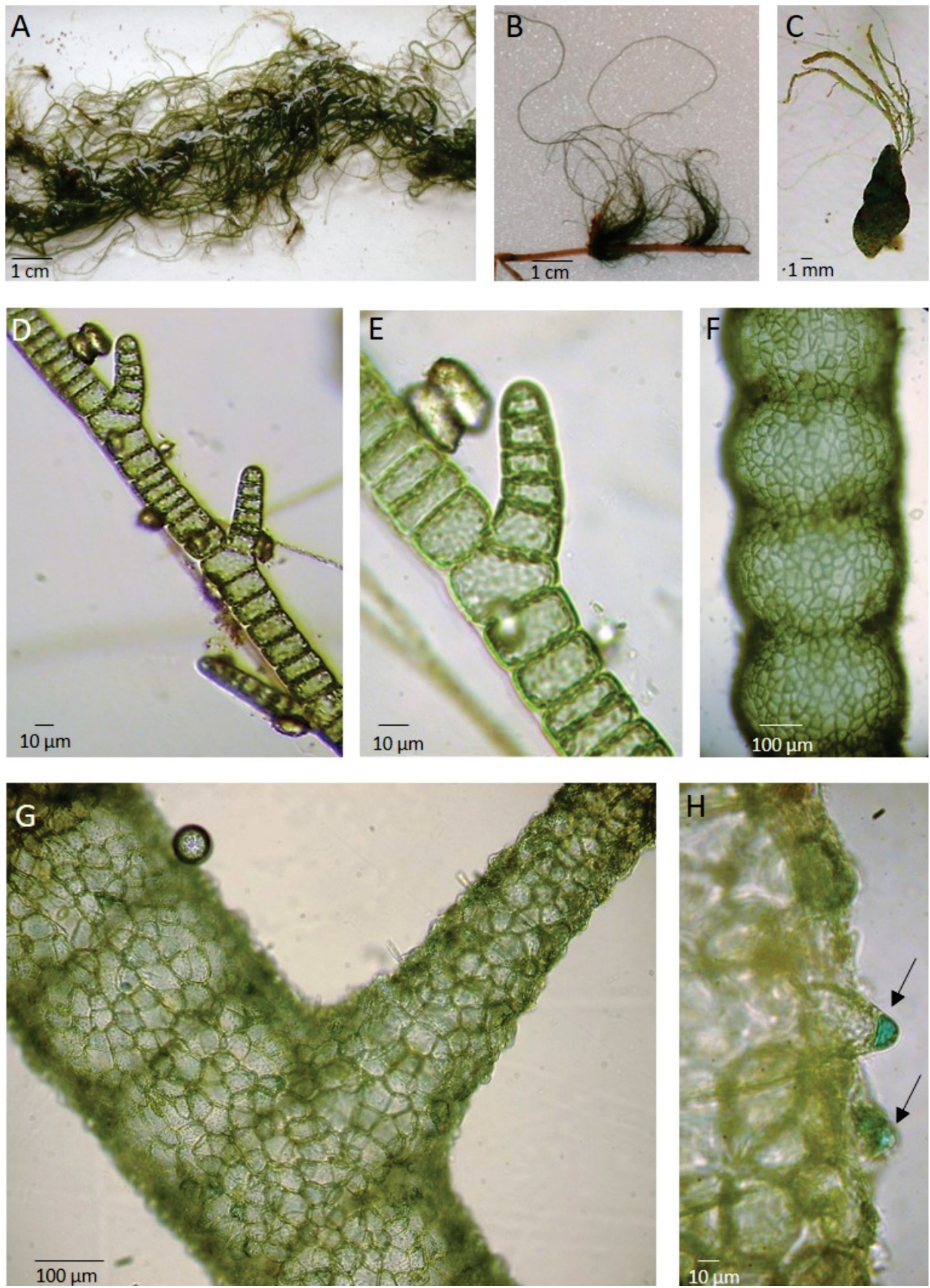

Fig. 3. Compsopogon caeruleus. A) Aspecto general. B) Epífito, sobre planta acuática. C) Epizoico, sobre caracol acuático. D) Ramificaciones uniseriadas. E) Detalle de ramificación uniseriada. F) Talo con constricciones anilladas. G) Talo con ramificación. H) Vista lateral de células corticales con monosporangios (flechas). 
la familia Poaceae, con dimensiones pequeñas (hasta $6 \mathrm{~cm}$ ) y coexistiendo con la rodofita Thorea sp. En los tres sitios se observó además la diatomea Pleurosira laevis entre los filamentos de Compsopogon.

Cabe mencionar que las algas que conviven con esta rodofita también han sido registradas por otros autores (Pandey, Tiwari y Pandey, 1976; Tracanna, 1979; Sabater et al., 1989; Leghari et al., 1997).

\section{DISTRIBUCIÓN}

A nivel mundial mencionada para todos los continentes (Guiry y Guiry, 2018). En Sudamérica citada en Brasil (Necchi, 1989). Debido a su habilidad para tolerar una amplia gama de condiciones ambientales (Necchi et al., 2013) es muy probable que esta especie posea una distribución más amplia en la provincia de Córdoba y en Argentina.

\section{MATERIAL ESTUDIADO}

ARGENTINA. Prov. Córdoba, Dpto. Capital, Lago del Parque Sarmiento, $31^{\circ} 25^{\prime} 53^{\prime \prime}$ S, 64010'44" O, 19-VI-2012, Leyes 130 (L.H.
UNC); 9-IX-2012, Leyes 131b (L.H. UNC); 9X-2014, Leyes 151 (L.H. UNC); Dpto. Colón, Arroyo Unquillo, $31^{\circ} 14^{\prime} 14^{\prime \prime} \mathrm{S}, 6^{\circ} 18^{\prime} 57^{\prime \prime} \mathrm{O}$, 2-VI-2013, Leyes 1 (PEA-CTUA INA); 30-V2014, Leyes 2 (PEA-CTUA INA); Dpto. Calamuchita, Río Tercero, 32 09'09" S, 6408'31" O, 4-V-2016, Leyes 3 (PEA-CTUA INA).

\section{OBSERVACIONES}

Históricamente, las características usadas para la delimitación de las especies del género Compsopogon incluyeron tipo de sistema basal, longitud del talo, patrón de ramificación, tamaño de los monosporangios, tamaño de las células corticales y número de capas corticales (Vis et al., 1992). Sin embargo, la mayoría de las especies reconocidas a nivel mundial son problemáticas a la hora de identificar debido a la superposición y variabilidad de esos atributos (Necchi et al.,1990; Vis et al., 1992; Rintoul et al., 1999). Además, estos organismos poseen una notable plasticidad fenotípica demostrada tanto en cultivo como en campo (Necchi et al., 2013). Como consecuencia de todo lo expresado,

Tabla 1. Caracteres morfológicos de las especies citadas en Argentina.

\begin{tabular}{|c|c|c|c|}
\hline \multirow[b]{2}{*}{ Caracteres } & \multicolumn{3}{|c|}{ Especies } \\
\hline & $\begin{array}{l}\text { Compsopogon } \\
\text { argentinensis }{ }^{* 1}\end{array}$ & $\begin{array}{l}\text { Compsopogon } \\
\text { occidentalis }{ }^{11}\end{array}$ & $\begin{array}{c}\text { Compsopogon } \\
\text { caeruleus } *^{2}\end{array}$ \\
\hline Longitud del talo $(\mathrm{cm})$ & hasta 26 & hasta 38 & hasta 60 \\
\hline Diámetro eje principal ( $\mu \mathrm{m})$ & 1200 & 1450 & 1500 \\
\hline Fijación al sustrato & $\begin{array}{l}\text { disco recubierto de } \\
\text { rizoides }\end{array}$ & rizoides & rizoides y/o disco \\
\hline $\begin{array}{l}\text { Células uniseriadas }(\mu \mathrm{m}) \\
\text { (longitud } \mathrm{x} \text { ancho) }\end{array}$ & $\begin{array}{c}13,2-36 x \\
3,2-22\end{array}$ & $\begin{array}{l}6-38 x \\
10-35\end{array}$ & $\begin{array}{c}20-29 x \\
16-20\end{array}$ \\
\hline Células corteza exterior $(\mu \mathrm{m})$ & $\begin{array}{l}13-46 x \\
10-30\end{array}$ & $\begin{array}{c}13-58 x \\
10-45\end{array}$ & $\begin{array}{c}20-57 x \\
16-37\end{array}$ \\
\hline Diámetro monosporangios $(\mu \mathrm{m})$ & $10-23$ & $10-18$ & $15-18$ \\
\hline № de capas de corteza & $1-3$ & $1-3$ & $1-2$ \\
\hline № de ejes & $1-6$ & 1-varios & 1- varios \\
\hline Forma de cromatóforos & oblongos, ovales & $\begin{array}{c}\text { cilíndricos, ovales, } \\
\text { esféricos }\end{array}$ & $\begin{array}{l}\text { acintados, } \\
\text { cilíndricos, } \\
\text { esféricos }\end{array}$ \\
\hline
\end{tabular}

${ }^{* 1}$ Extraído de Pujals (1967) y Tracanna (1979) respectivamente.

${ }^{*}{ }^{2}$ Los valores corresponden a los registrados en este trabajo. 
desde el establecimiento del género han existido controversias acerca del número de especies incluidas en el mismo.

En un estudio Vis et al. (1992) cotejaron los caracteres morfológicos proporcionados en las descripciones originales de diferentes especímenes tipo (entre ellas C. argentinensis), y no pudieron establecer una clara diferenciación con respecto a $C$. caeruleus. Necchi y Pascoaloto (1995) demostraron que estos caracteres son ampliamente variables y tienen escaso valor taxonómico. En la Tabla 1 se presentan las características de las especies citadas en Argentina y la del presente estudio.

Por otra parte, en una investigación de Necchi et al. (2013) se compararon a nivel genético 24 ejemplares procedentes de diferentes continentes (exceptuando Asia), identificadas en principio como especies distintas. En base al análisis molecular llegaron a la conclusión que el género posee poca variación genética y sería monoespecífico, de amplia distribución mundial y plasticidad morfológica considerable. Consecuentemente, proponen que todas las especies descriptas debieran ser consideradas sinónimos de C. caeruleus. Sin embargo, en estos análisis no se evaluaron individuos encontrados en Argentina, por lo tanto serían necesarios nuevos estudios a fin de dilucidar la identidad taxonómica de las especies encontradas en el país.

\section{CONCLUSIÓN}

Este trabajo brinda información sobre un género escasamente estudiado y registrado en Argentina y permite a su vez ampliar la riqueza de macroalgas en la Provincia de Córdoba.

\section{BIBLIOGRAFÍA}

Bourrelly, P. (1970). Les algues d'eau douce. Initiation á la systématique. Tome III: Les Algues bleues et rouges. Les Eugléniens, Peridiniens et Cryptomonadines. Paris: Boubée Cie.

Carmona Jiménez, J. y Beltrán Magos, Y. P. (2007). Las algas rojas de aguas continentales en la región central de México.
México: Universidad Nacional Autónoma de México.

Ceschin, S., Ricci, S., Abati, S., Bisceglie, S, Minciardi, M. R. y Zuccarello, V. (2013). Distribution and ecology of red algae in Italian rivers. Fundamental and Applied Limnology 183: 223-237. doi: $10.1127 / 1863$ - 9135/2013/0493.

Daga, C., Soteras, F., Daniele, G. M. y Dominguez, L. S. (2014). New records of freshwater Algae and Cyanobacteria from mountain streams of Córdoba (Argentina). Boletín de la Sociedad Argentina de Botánica 49 (4): 447-456.

Gauna, M. C. y Parodi, E. R. (2005). Morfología y ciclo de vida de Batrachospermum atrum var. puiggarianum (Batrachospermales, Rhodophyta). Darwiniana 43 (1-4): 57-63.

Guiry, M. D. (2012). How many species of algae are there?. Journal of Phycology 48, 1057-1063. doi: 10.1111/j.15298817.2012.01222.x.

Guiry, M. D. y Guiry, G. M. (2018). AlgaeBase. World-wide electronic publication, National University of Ireland, Galway. Recuperado de http://www. algaebase.org.

Krishnamurthy, V. (1962). The morphology and taxonomy of the genus Compsopogon Montagne. Journal of the Linnean Society [Botany) 58: 207-222.

Lazo, M. L. (1978). El ciclo biológico de Batrachospermun puiggarianum Grunow (Rhodophyta). (Tesina de grado). Facultad de Ciencias Exactas Físicas Naturales, Universidad Nacional de Córdoba.

Leghari, S. M., Sahito, G. N., Hayee-Memon, A., Khuhawar, M. Y. y Mastoi, G. M. (1997). Freshwater red algae in water effluents of thermal power house at Jamshoro, Sindh, Pakistan. Pakistan Journal of Botany 29 [1]: 151-160.

Leyes, C. (2016). Contribución al conocimiento taxonómico de cianobacterias y algas del lago del Parque Sarmiento (Córdoba, Argentina). (Tesina de grado). Facultad de Ciencias Exactas Físicas Naturales, Universidad Nacional de Córdoba.

Leyes, C., Falco, C. y Daga, C. (2018). Aporte al conocimiento de la ficoflora del lago del Parque Sarmiento [Córdoba, Argentina). Revista de la Facultad de Ciencias Exactas, Físicas y Naturales 5 (1): 45-50.

Liu, S. L. y Wang, W. L. (2004). Two New Members of Freshwater Red Algae in Taiwan: Compsopogon tenellus Ling et Xie and C. chalybeus Kützing (Compsopogonaceae, Rhodophyta). Taiwania, 49 (1): 32-38. 
Montagne, J. F. M. (1846). Algae. En: M. C. Durieu de Maisonneuve, J. F. C. Montagne, J. B. G. M. Bory de Saint-Vincent, L. R. Tulasne, C. Tulasne, J. H. Leveille [Eds], Flore d'Algerie Cryptogamie, premiere partie. Paris : Imperiale.

Nan, F., Feng, J., Lv J., Liu, Q., Fang, K., Gong, C. y Xie, S. (2017). Origin and evolutionary history of freshwater Rhodophyta: further insights based on phylogenomic evidence. Scientific Reports 7: 2934. doi:10.1038/s41598-01703235-5 1

Necchi, 0. (1989). Rhodophyta de água doce do Estado de São Paulo: levantamento taxonômico. Boletim de Botanica, Universidade de São Paulo 11: 11-69.

Necchi, O., Góes, R. M. y Dip, M. R. (1990). Phenology of Compsopogon coeruleus (Balbis) Montagne (Compsopogonaceae, Rhodophyta) and evaluation of taxonomic characters of the genus. Japanese Journal of Phycology 38: 1-10.

Necchi, O. y Pascoaloto, D. (1995). Morphometry of Compsopogon coeruleus (Compsopogonaceae, Rhodophyta] populations in a tropical river basin of southeastern Brazil. Algological Studies 76: 61-73.

Necchi, O., Branco, C. C. Z. y Gomes, R. R. V. (1999). Microhabitat and plant structure of Compsopogon coeruleus (Compsopogonaceae, Rhodophyta) populations in streams from São Paulo State, southeastern Brazil. Cryptogam Algol 20: 75-87.

Necchi, O., Fo, A. S. G., Salomaki, E. D., West, J. A., Aboal, M. y Vis, M. L. (2013). Global sampling reveals low genetic diversity within Compsopogon (Compsopogonales, Rhodophyta). European Journal of Phycology 48 (2): 152162. doi: 10.1080/09670262.2013. 783626
Pandey, R. S.,Tiwari, G. L. y Pandey, D. C. (1976). Observations on Compsopogon iyengarii Krishnamurthy (Rhodophyceae). Hydrobiologia 49.3: 239-244.

Pujals, C. (1967). Presencia en Argentina de Compsopogon (Rhodophycophyta, Bangiophyceae). Comunic. Museo Argent. Ci. Nat. B. Rivadavia, Ser. Hidrobiol. 1: 47-53.

Rintoul, T. L., Sheath, R. G. y Vis, M. L. (1999). Systematics and biology of the Compsopogonales (Rhodophyta) with emphasis on the freshwater families in North America. Phycologia 38 (6): $517-$ 527. doi: 10.2216/i0031-8884-38-6517.1.

Sabater, S., Aboal, M. y Cambra, J. (1989). Nuevas observaciones de rodofíceas en aguas epicontinentales del NE y SE de España. Limnética 5: 93-100.

Sánchez Rodríguez, M. E. (1974). Rodofíceas dulceacuícolas de México. Boletín de la Sociedad Botánica de México 33: 31-37.

Sheath, R. G. y Hambrook, J. A. (1990). Freshwater ecology. En: K. M. Cole, R. G. Sheath (Eds.), Biology of the red algae (pp. 423-453), New York: Cambridge University Press.

Taboada, M., Martínez de Marco, S. y Tracanna, B. (2016). Biodiversidad epilítica de un arroyo subtropical del Noroeste Argentino. Lilloa 53 (1): 122-132.

Tracanna, B. C. (1979). Contribución al conocimiento de las algas del Noroeste Argentino, II Compsopogon occidentales sp. nov. (Rhodophyta). Lilloa 35 (2): 35-37.

Vis, M. L., Sheath, R. G. y Cole, K. M. (1992). Systematics of the freshwater red algae family Compsopogonaceae in North America. Phycologia 31 (6): 564575. doi: 10.2216/i0031-8884-31-6564.1.

Wehr, J. D. y Sheath, R. G. (2003). Freshwater Algae of North America. Amsterdam: Academic Press. 